\title{
USE OF SIMULTANEOUS INTEGRATED BOOST INTENSITY-MODULATED RADIATION THERAPY TECHNOLOGY IN LARYNGEAL CARCINOMA
}

\author{
Muhammad Abdul Rafaye, Sadaf Usman, Arif Jamshed \\ Department of Radiation Oncology, Shaukat Khanum Memorial Cancer Hospital and Research Centre, Lahore, \\ Pakistan
}

Received: 03 March 2017 / Accepted: 28 March 2017

Head and neck cancer is one of the most common cancers in Pakistan. Most of the patients present in advance stage. Laryngeal cancers account for $13 \%$ of head and neck cancer patients in our institution. Most of the advance laryngeal cancer patients treated with chemoradiation have poor quality of life due to poor function of the preserved larynx, ultimately needing salvage laryngectomy. Therefore, total laryngectomy followed by adjuvant radiotherapy remains the standard of care in T4 laryngeal cancer patients. Conventional radiotherapy techniques result in increased acute as well as long-term toxicity which impairs the quality of life, note ably xerostomia. ${ }^{[1]}$

Simultaneous integrated boost intensity-modulated radiation therapy (IMRT) is an advance radiotherapy technique allowing sparing of organs at risk (OAR) while giving high and homogenous dose to the primary target. It allows shorter overall treatment time with hypofractionation and dose escalation to primary disease with high biological equivalent dose (BED). In developing countries, it serves to spare the resources in terms of equipment usage in shorter fractionations, providing equivalent local control with reduced toxicity profile. ${ }^{[2,3]}$

A case of laryngeal carcinoma treated with total laryngectomy followed by adjuvant radiotherapy of $60 \mathrm{~Gy}$ in 30 fractions was treated with simultaneous integrated boost IMRT technique. A 66-year-old male patient presented to an ENT clinic with an 8-month history of hoarseness of voice. Biopsy revealed well-differentiated squamous cell carcinoma. Staging computed tomography (CT) neck with contrast showed glottic tumour associated with supra and subglottic extension. There was no regional lymphadenopathy. The patient was planned for total laryngectomy. Histopathology showed moderately

Correspondence: Muhammad Abdul Rafay, Department of Radiation Oncology, Shaukat Khanum Memorial Cancer Hospital and Research

Centre, Lahore, Pakistan. Email: arafaye@skm.org.pk differentiated squamous cell carcinoma, $3.5 \mathrm{~cm}$ in size, invading through thyroid cartilage and focally invaded soft tissue of neck (pT4a), margins free of tumour, no lymphovascular invasion or perineural invasion. No subglottic extension seen. 1 of 47 lymph nodes was positive. He had nutritional and dental assessment before radiotherapy.

CT for radiotherapy treatment planning was done with patient positioned supine, immobilized with a thermoplastic mask with neck in extended position. CT images of $3 \mathrm{~mm}$ slice thickness were acquired from vertex to carina. The clinical target volume was drawn on each CT slice using information from pre-operative imaging, operation notes and discussion with surgeon including sites at risk of microscopic disease. The CTV50 included areas of subclinical disease -the bilateral level Ib-V neck nodes. CTV60 included the tumour BED alone. PTV50 and PTV60 were formed by giving $5 \mathrm{~mm}$ margin to CTV50 and CTV60, respectively, as shown in Figures 1 and 2. OARs outlined included the brainstem, spinal cord, parotid glands, bilateral temporomandibular joint (TMJ) and cochlea.

Simultaneous integrated boost IMRT technique was used for treatment planning. PTV60 received 2.0 Gy daily dose up to 30 fractions while PTV50 received a daily dose of 1.8 Gy up to 30 fractions. The dose constraints for OARs were as follows: 40 Gy maximum point dose to brainstem, maximum point dose of $40 \mathrm{~Gy}$ to spinal cord, mean dose $<30$ Gy to each parotid, mean dose of $<50$ Gy to cochlea and mean dose $<60$ Gy to TMJ. Inverse planning was performed using Varian Eclipse Treatment Planning Software Version 11. This is shown in Figures 1-3.

Treatment plan was evaluated using dose-volume histograms and found the doses as follows: $95 \%$ of the prescribed dose $(57.8 \mathrm{~Gy})$ to PTV60, $100 \%$ of the prescribed dose (50 Gy) to PTV50, maximum dose of 


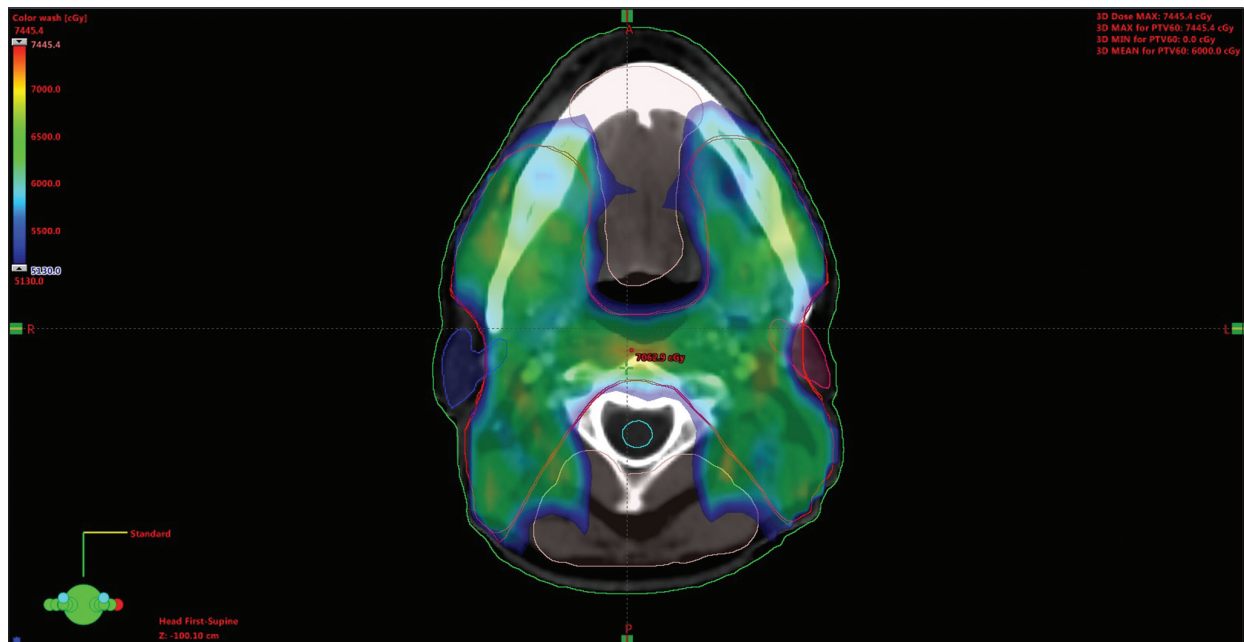

Figure 1: Axial CT image of the neck showing CTV60 (tumour bed only) and CTV50 (sub-clinical disease and neck nodes)

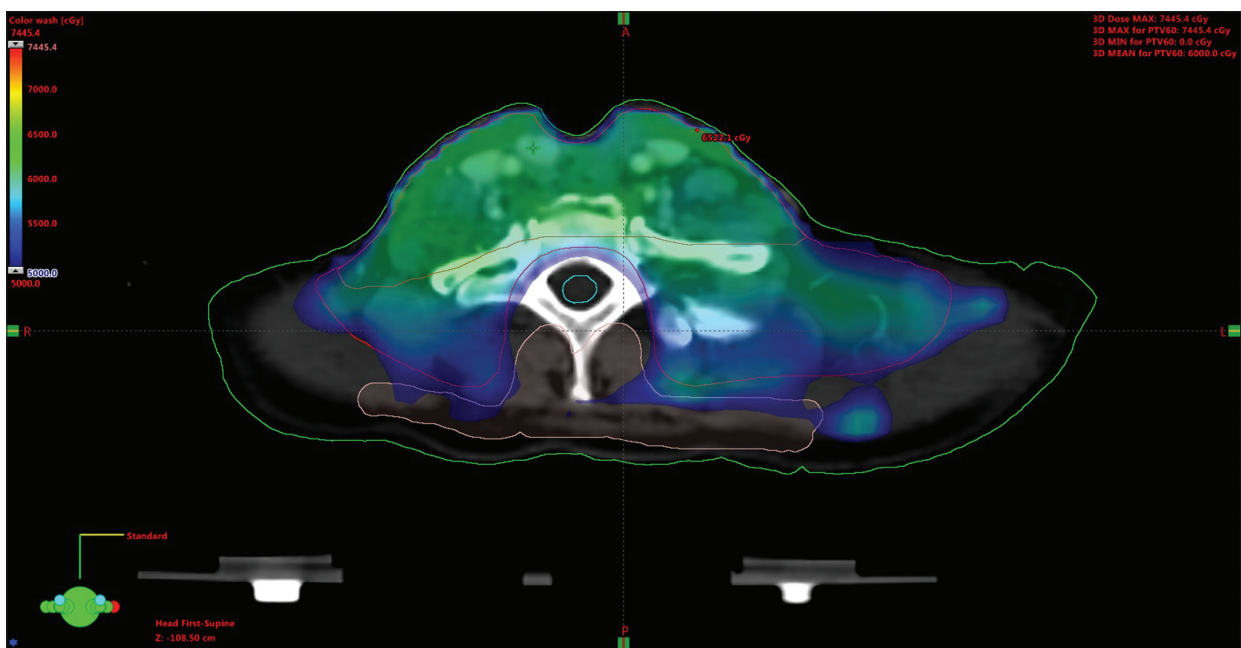

Figure 2: Axial CT image of lower neck with PTV60 (3mm margin to CTV60)

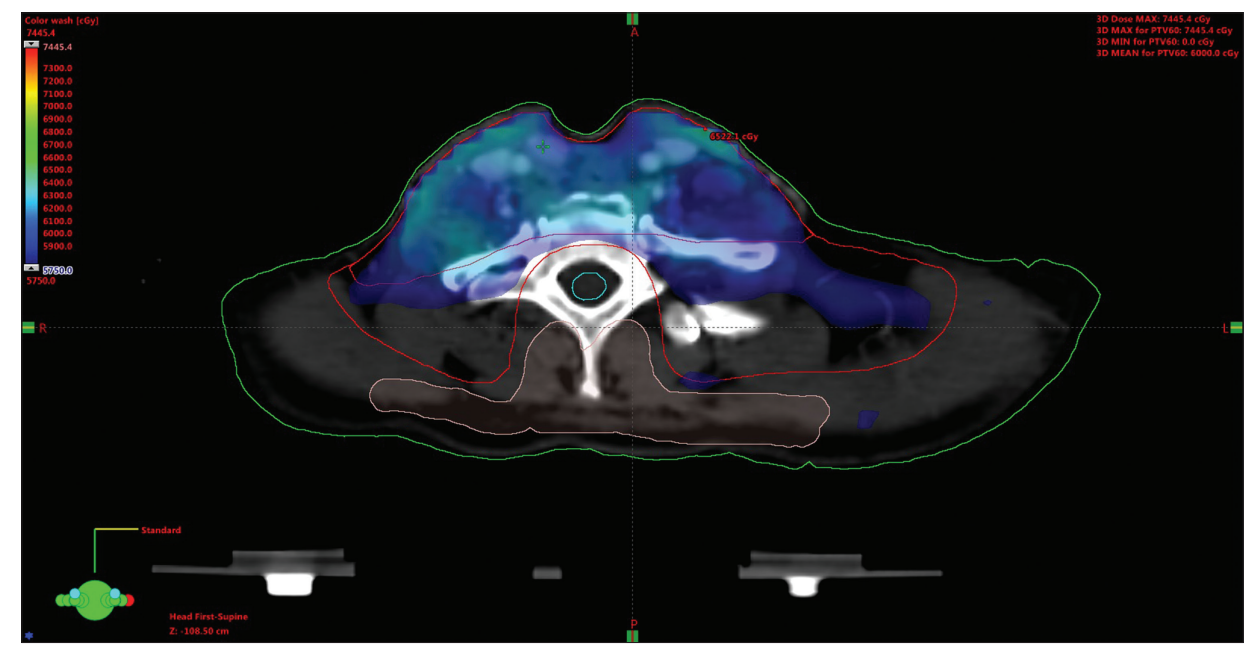

Figure 3: Axial CT image of lower neck with PTV50 (3mm margin to CTV50) 
41 Gy to brainstem, maximum point dose of $43 \mathrm{~Gy}$ to spinal cord and mean dose of 26 Gy to parotid.

This patient completed the treatment in 6 weeks without any interruption weekly clinical assessment was done during the treatment for this patient, and he was assessed for the acute toxicity defined as side effects occurring during radiotherapy. This assessment included the grading of side effects according to common toxicity criteria version 3. He was assessed in the clinic at 6-week post radiotherapy. Acute skin toxicity and mucositis had settled and there was no xerostomia. Physical examination revealed no local or regional recurrence.

This concludes that simultaneous integrated boost intensitymodulated radiotherapy technique allowed the treatment to be completed with higher doses to the target volumes sparing OAR with reduced acute toxicity profile. The patient returned to a good quality of life early with no xerostomia.

\author{
Abbreviations: \\ CT: Computed tomography \\ CTV: Clinical Tumour Volume receiving 50 or 60 grays
}

DVH: Dose volume histograms

IMRT: Intensity modulated radiation therapy

OAR: Organ at risk

PTV: Planning Tumour Volume receiving 50 or 60 grays

TMJ: Temporomandibular joint

\section{Conflict of Interest}

The authors declare that they have no conflicts of interest.

\section{References}

1. Deodato F, Cilla S, Macchia G, et al. IMRT/VMATSIB technique chemoradiation in locally advanced head and neck cancer: Toxicity results. Radiother Oncol 2016;119:S501-2.

2. Pyakuryal A, Pandit S, Narayanasamy G, et al. Radiobiologic evaluation of simultaneously integrated boost (SIB) IMRT methods in head and neck cancer: Multi-institutional study. Int J Radiat Oncol Biol Phys 2014;90:S789-90.

3. Leclerc M, Maingon P, Hamoir M, et al. A dose escalation study with intensity modulated radiation therapy (IMRT) in T2N0, T2N1, T3N0 squamous cell carcinomas (SCC) of the oropharynx, larynx and hypopharynx using a simultaneous integrated boost (SIB) approach. Radiother Oncol 2013;106:333-40. 